\title{
Pospandemia y bibliotecas: ¿y ahora qué?
}

\section{( Alejandro E. Parada \\ Secretario de Redacción / Información, cultura y sociedad Universidad de Buenos Aires, Facultad de Filosofía y Letras, Instituto de Investigaciones Bibliotecológicas-INIBI, Argentina | aparada@filo.uba.ar / https://orcid.org/oooo-0002-2331-442X}

\begin{abstract}
Resumen
En este Editorial se reflexiona sobre varios aspectos que, posiblemente, pueden presentarse en las bibliotecas en un escenario de pospandemia. Aunque la bibliografía sobre esta temática ya es abundante e incontrolable y, además, se corre el riesgo de caer en una prospectiva bibliotecaria que exceda la realidad, el Editorial intenta analizar varios tópicos de interés profesional para las bibliotecas: la necesidad de adaptarse a los nuevos tiempos con el objetivo de supervivir, la adecuación de un equilibrio entre lo remoto y presencial, la profesionalización de la vida doméstica, la encrucijada de estas instituciones para reconvertirse en lugares de enseñanza, las nuevas alianzas de bibliotecas con fines pedagógicos, la importancia de reconfigurar la identidad gremial de sus profesionales, entre otros temas. Así, dentro de este contexto, la pospandemia constituye un desafío para afianzar el crecimiento de las bibliotecas y para promover nuevos derechos de ciudadanía.
\end{abstract}

\section{Post-pandemic and libraries: what now?}

Palabras clave

\section{Bibliotecas} Pospandemia Bibliotecología y Ciencia de la Información

\begin{abstract}
The following editorial analyzes some aspects that may possibly occur in libraries in a post-pandemic setting. Although the bibliography on this subject is already abundant and uncontrollable and, in addition, there is a risk of falling into a librarian prospective that exceeds reality, the Editorial attempts to analyze various topics of professional interest to libraries: the need to adapt to the new times in order to survive, the adequacy of a balance between the remote and the face-toface, the professionalization of domestic life, the crossroads of these institutions to convert themselves into teaching places, the new alliances of libraries with pedagogical purposes, the importance of reconfiguring the union identity of its professionals, among other issues. Thus, within this context, the post-pandemic constitutes a challenge to consolidate the growth of libraries and to promote new citizenship rights.
\end{abstract}

\section{Keywords}

$\overline{\text { Libraries }}$

Post-pandemic

Librarianship and Information Science 
Las bibliotecas en la actualidad, acaso más que nunca, se encuentran en un dilema de conflicto acerca de sus modos y prácticas de existencia. En esta afirmación no hay ningún hallazgo. Las "vicisitudes de permanencia" las han acompañado desde siempre. Guerras, desastres naturales, cambios políticos, disponibilidad exigua de recursos, desidia, enfrentamientos religiosos, entre muchos acontecimientos y avatares, las han asediado constantemente hasta convertirse en el ADN de su propia dinámica. No hay identidad bibliotecaria sin trance ni desequilibrio. En cierto sentido, aunque esta aproximación connote una arista paradojal, la matriz de esas encrucijadas -signadas por "el riesgo constante"- también ha devenido en el impulso y afianzamiento de estas instituciones sociales. Porque la "resistencia y la supervivencia" es una de sus más notables propiedades, con una larga y continua tradición histórica.

Antes de la pandemia, las bibliotecas estaban adaptándose con éxito a una de las mayores revoluciones del libro: su renovación y mutación material. El desafío se centraba -y ahora con mayor intensidad- en reconvertir, según el tipo de unidades, buena parte de las colecciones impresas en archivos digitales para su lectura en diferentes dispositivos. Esta delicada operación relacionada con la transformación de la materialidad tipográfica demandaba una planificación y, específicamente, un abordaje proporcionado entre el universo impreso y el digital. Una situación que debía acompañar a los paulatinos cambios de los procedimientos de lectura (Chartier, 2021). Pero con la pandemia, todo emprendimiento de llevar a cabo esa transición en forma ordenada ha resultado, por lo menos, limitado o puesto entre paréntesis ante una coyuntura impensada que ha afectado a la mayoría de los estamentos de la sociedad y, ciertamente, a la dispersión y a la continuidad de los servicios de las bibliotecas.

A esto hay que agregar, la intensa readecuación bibliotecaria de perfiles conceptuales de gran significación. Un claro ejemplo de este salto epocal, y que será determinante en la pospandemia, es el pasaje, tanto de estudiantes, docentes, investigadores y usuarios en general, de ser definidos como "visitantes digitales a residentes digitales" (Connoway, 2020). No es lo mismo, pues, residir ocasionalmente en las bibliotecas que ser, diariamente, un constante residente digital.

Ante todo, el desafío para garantizar la sobrevivencia tiene su epicentro en la extraordinaria adecuación que poseen las bibliotecas para afrontar la heterogeneidad de los cambios a las que han estado sometidas, aun de aquellas mutaciones que no habían sido nunca concebidas (ABGRA, 2020; Gutiérrez y Castaño, 2020; INIBI, 2020). Por eso se plantea (ya lo han hecho una gran cantidad de colegas) qué sucederá después de la pandemia cuando la apertura condicionada sea un acontecimiento impuesto bajo el umbral de la pospandemia. Entonces, el ¿y ahora qué? se volverá (ya es) en un nuevo reto cuyas consecuencias resultan de compleja dilucidación.

La pandemia modeló el futuro bibliotecario hasta comprimirlo en una realidad inmediata, en un ubicuo presente marcado por una vertiginosa atemporalidad. Y también propició una especie de coda aleatoria, en su regreso y proyección a ese mismo presente en suspensión. Resulta ocioso y redundante explayarnos en este tópico, pues la literatura sobre bibliotecas y COVID-19 es tan copiosa, en tan solo el fatídico año 2020 , que prácticamente desalienta cualquier intento de acercamiento sistemático. Por eso este Editorial solo aborda unos mínimos aspectos, de matiz personal y profesional, relacionados con su ya ingobernable bibliografía representada por cientos de contribuciones (una simple búsqueda de "bibliotecas y pandemia" es una experiencia abrumadora).

A partir de este marco, las modalidades remotas y las videollamadas se aceleraron con una exigencia desbordada y, muchas veces, con pocas posibilidades de implementar criterios de normalización en sus procederes. Pues la pandemia, en el universo 
bibliotecario, trajo consigo un profundo desorden en la ejecución de la gestión, aún en las menores actividades administrativas. El acceso ordenado y normativo característico del mundo presencial se metamorfoseó en una presentización casi caótica del propio devenir de las bibliotecas. Pero el verdadero tema en cuestión, y que nadie puede determinar con certeza sus límites móviles, es la maleabilidad de nuestra mentalidad bibliotecaria. Un presente a caballo entre lo presencial y lo remoto, entre lo impreso y lo digital e inmerso en una virtualidad que, aunque esperada, ya se ha precipitado en un escenario definitivo.

La adaptación del pensamiento bibliotecario es una función nucleada en la capacidad de reconocer, observar, describir y habitar, con un espíritu práctico creador, en el impetu arrollador de esta impostergable "galaxia remota"; esto es, de un "mundo feliz" que supera a la Galaxia Gutenberg. No solo navegamos a través de las bibliotecas y sus colecciones sino, además, en la marea de nuestras ideas y mentalidades que intentan reajustarse a lo que hoy esperamos de las bibliotecas y de su respuesta pospandémica. Formamos parte de esa única corriente, de un flujo entre el estar y el deber ser como bibliotecarios y bibliotecarias. La biblioteca adquiere así, en la presentización de su futuro, una realidad cognitiva simbólica y fronteriza. Lo nodal se centra en establecer los márgenes indeterminados de sus fronteras, donde asistimos, por añadidura, a una plena colonización de los servicios frente a las demandas digitales y remotas. Un proceso que ya estaba en marcha y que la pandemia fue, ni más ni menos, un acelerador del destino, en gran parte a distancia, de las bibliotecas. Un destino mediado y conformado por un elemento insoslayable: "lo distante".

¿Cuáles son, pues, nuestros márgenes de identidad más allá de la pandemia y qué desconocidos territorios y geografías nos aguardan en los tiempos de pospandemia bibliotecaria? Una pregunta sin respuestas unívocas y con múltiples interrogantes encerradas en un laberinto. De todos los pensamientos aparentemente perdidos en un dédalo ¿cuál es el que salta por encima del cerco y encuentra el camino de la razón? Porque el pensamiento racional debe buscar un procedimiento vivencial al aislamiento individual y autosuficiente de la seducción remota.

Porque en "los ahora qué", la innovación imaginativa no será algo sesgado u oblicuo. ¿Qué haremos de las bibliotecas en ese luego de la pandemia? Un "pos-después" que también involucra su articulación con los espacios de circulación anteriores a esta emergencia. Porque lo impreso y su manipulación material aún vive y palpita como una maravilla por descubrir. Es oportuno priorizar, indudablemente, todos los procesos de digitalización dentro de un objetivo bifronte; por un lado, gestionar las colecciones desde sus ámbitos tradicionales bibliotecarios; luego, en un segundo momento determinante, diseñar los servicios presenciales desde nuestros hogares (no como una excepcionalidad de la pandemia), si no como un itinerario real en la pospandemia. Hacer de la profesionalidad, una ida y vuelta de lo institucional a lo doméstico y de este último al primero. El desarrollo del trabajo profesional tiene así un renovado sesgo económico (en su amplio sentido administrativo) intermediado por las conductas profesionales. La línea divisoria entre lo profesional y la vida doméstica comienza a acentuar su difusión e invisibilidad, como la indecisa vida pública y privada que llevamos en las redes sociales. En una amplia e indefinida travesía, acontece la profesionalidad en lo cotidiano y, en consecuencia, la institucionalidad de las bibliotecas debe ser posicionada a partir otra esfera.

La biblioteca, en sí misma, deja de ser una institución tal como la conocimos, para convertirse en una entidad por definir, en una institución o agencia que requiere de una refundación conceptual y programática, fuera de su ensimismamiento en las conductas heredadas. Un nuevo suceder de índole atemporal en ese manifiesto presente que instaura el dominio de los firmamentos remotos y digitales. Estamos hablando 
de una filosofía moral, individual, colectiva e institucional diferente de aquello que conocimos como "biblioteca", o de aquello que en cuanto personas identificábamos con los lugares donde estas se desplegaban. La pospandemia enlazará, en una vertiente de alta complejidad, nuestro hacer diario con los restos institucionales que aún perduran y que requerirán de una reestructuración. ¿Qué itinerarios y virajes tendrá, en este caso, el brío de la imaginación enlazada con la racionalidad para desinstitucionalizar los formalismos bibliotecarios que nos han caracterizado hasta la fecha?

Pero también se impone una pregunta: ¿nos postraremos, sin reticencias algunas, ante el Dios de la digitalización y sus solicitudes a distancia? ¿Haremos de lo remoto y la virtualidad una divinidad aceptada e inamovible? Detrás de todo momento diferido hay una nostalgia y una necesidad por "la naturaleza de lo natural". El artificio de lo remoto oculta un clamor: la necesidad del encuentro personal. Los encuentros en línea simultáneos tanto en interacción sincrónica o no, fragmentan nuestra imagen en una postergación de lo humano, liderado por la retórica facetada de las teleconferencias. A la fragmentación de las prácticas lectoras sobre los textos digitalizados y sus usos de lectura en distintas unidades electrónicas, le corresponde un incremento parcelado de las bibliotecas. Así también emerge un profesional fraccionado y suspendido temporalmente, con diversos accesos mediados y distantes. De este modo, si en la pospandemia las reuniones con los usuarios aún serán preponderantemente remotas, se torna de vital importancia rescatar, mediante todo tipo de estrategias, una revalorización de la esencialidad presencial. Por supuesto, no nos referimos a un intercambio cara a cara, pleno y rotundo, de los lectores en las bibliotecas. Nos referimos a instrumentar no solo los protocolos de sanidad indispensables sino, además, a tender los lazos para la reconstrucción de ese vínculo de la intimidad que pauta a todo encuentro personal. Una esencia gregaria que no debemos perder bajo el imperio de lo remoto, para no ser solo "profesionales en tránsito".

Tras la condición de comunidad inmersa en la biblioteca, subyace y alienta el humanismo bibliotecario. Nuestro diálogo pospandémico deberá desentrañar el lenguaje fundacional entre lo remoto y lo presente. El punto de inflexión radica, posiblemente, en redefinir nuestra actitud humanística y tecnológica, pero ahora rediseñada por el ímpetu y la realidad virtual. En estas circunstancias, se presenta la posibilidad y la necesidad de una reforma bibliotecaria de nuestros hábitos presenciales, precisamente para rescatar el valor impostergable de "lo presencial". ¿Qué tipo de reforma necesitamos, qué tipos de cambios se podrán instrumentar para garantizar una mínima tangibilidad del rostro de nuestros lectores y lectoras? Esta es una de las disyuntivas más acuciantes de las bibliotecas en la pospandemia, una vuelta de tuerca inesperada (pero no menos veraz) de la presencia en el vórtice de un pos-posmodernismo. Porque, al final de cuentas, todo se dirimirá en el impacto de nuestra actitud parapresencial.

Sin embargo, independientemente de los proyectos que adoptemos en el universo de interacción y acoplamiento entre lo impreso y lo digital, hay otras particularidades a tener en cuenta. Las arquitecturas bibliotecarias tendrán que reconfigurar sus dimensiones espaciales (los espacios o salas de lectura tradicionales) a lugares de enseñanza y aprendizaje, sea presencial o a distancia (Suarez, 2020). Esto ya está sucediendo hace varios años y existe una abundante cantidad de trabajos que lo desarrollan desde ángulos distintos. El profesional remoto necesita incrementar el contexto social de las bibliotecas. En este punto es significativo reflexionar sobre el aspecto pedagógico que involucra a los y las bibliotecarias en esa nutricia migración de las salas de lectura a las aulas de enseñanza. La profesión, para supervivir y trasmitir la cultura del libro y sus prácticas de lectura, deberá migrar, en forma decidida, a una experticia vinculada con la instrucción. En este contexto, la preservación de las bibliotecas y sus acervos impresos y digitales, dependerá de nuestro desarrollo pedagógico y de su incidencia en la sociedad. Los planes de estudios de las carreras 
de Bibliotecología y Ciencia de la Información tendrán que adecuarse a un profesional que satisfaga estas nuevas y perentorias necesidades (Gaitán y Coraglia, 2021; Arroyo-Vázquez y Gómez-Hernández, 2020). Hay, pues, que prepararse para enseñar desde y para las bibliotecas.

Aunque resulta dificultoso saber cómo se desarrollará luego del Coronavirus este proceso de enseñanza-aprendizaje en las bibliotecas, es de real interés volver a conceptualizar una práctica bibliotecaria de las últimas décadas: la solidaridad y el entramado de las redes de bibliotecas y las modernas alianzas institucionales. Para afianzar y amplificar sus herencias culturales como agentes activos, hay que generar una trazabilidad que posea contundencia y no caiga en una simple aspiración voluntarista. Será indispensable una fortaleza conjunta de los diversos tipos de bibliotecas y, por consiguiente, una sustentabilidad sostenida en el tiempo acerca de sus posibilidades educativas. Un objetivo que deviene en alianzas novedosas con otras instituciones pero mediadas por el empoderamiento de las redes interbibliotecarias. Redes que ya no solo se fundamenten en la adquisición cooperativa de materiales impresos o paquetes digitales, o en otros requerimientos materiales, sino que también impulsen a las bibliotecas como campos de educación curricular. Una controversia con sesgos casi épicos, pues ganarse un sitial de reconocimiento en la educación formal, por supuesto, constituirá una polémica que debe encontrarnos a todos los profesionales unidos, y que solamente estaría a nuestro alcance al reconfigurar los objetivos de las redes de cooperación bibliotecaria que, tras este supuesto, terminará por ser una de las formas de sobrevivencia de las bibliotecas.

No obstante, a pesar de que las videollamadas encubren una limitación del intercambio presencial y que proyectan la virtualidad como "un espejismo de la realidad", con sus más y sus menos en cuanto a sus grandes beneficios, traerán aparejado otros contextos más dramáticos del punto de vista social de nuestra profesión. Tal como sucedió con los inicios de la revolución industrial y la introducción de las máquinas a vapor en sustitución del trabajo manual, el trabajo remoto cuestionará la necesidad de numerosos puestos de trabajo en las bibliotecas. El "trabajador remoto", si bien se realizaron algunos intentos aún tempranos por legislar su situación, estará expuesto tanto al recorte de los derechos laborales presenciales como a la precariedad de las condiciones a distancia. La mixtura entre lo institucional y lo doméstico que hemos arriba mencionado, no es otra cosa que una nueva legislación del trabajo y del trabajador. Porque nuestra realización práctica, aunque a veces no exista una plena conciencia de ello, es una realización profesional vinculada a las leyes laborales que regulan sus derechos.

No resulta extraño o irreal, quizás más en el sector privado que en el público, que la presencialidad de las personas en sus hogares migre hacia la condición de "personas prescindibles", en un duro e irreparable juego de palabras. Es decir, la expulsión de muchos y muchas de nuestros colegas de sus trabajos. Este marco de pospandemia, entonces, puede ser una oportunidad para que los "dueños del trabajo" tengan la oportunidad de vaciar y pauperizar a una gran cantidad de fuentes laborales. Ante la complejidad de este cuadro, el riesgo subyace en la infravaloración de dicho panorama. Todo hace pensar que la continuidad y legitimidad de gran cantidad de profesionales dependerá de una mayor participación de las funciones gremiales bibliotecarias, con el objetivo insoslayable de defender las amenazas que se ciernen sobre nosotros y nosotras.

Otro de los tópicos clave que se manifestó durante la pandemia fue la discusión social (y también existencial) sobre los derechos civiles y humanos a la libre e irrestricta información que brindan las bibliotecas. Durante el transcurso de la cuarentena y, posteriormente, con la implantación del distanciamiento preventivo social y obligatorio en 
la Argentina, entre muchos países, la totalidad de las bibliotecas tuvieron que cerrar sus puertas y luego, paulatinamente, además de ciertos servicios remotos, brindar un mínimo acceso a sus colecciones mediante protocolos y normativas. Se realizaron y realizan experiencias de todo tipo y, algunas de ellas, con un gran impacto en sus usuarios, como sucedió en la provincia de Mendoza (Rodríguez, 2020), entre numerosos ejemplos (Leer la ciudad, 2020; Las bibliotecas populares, 2020).

Pero este tema pone en escena una propiedad determinante y siempre impostergable de las bibliotecas: son instituciones que construyen ciudadanía y que proyectan los derechos de igualdad ante la ley en toda democracia, al menos en la letra y en el espíritu de las constituciones de cada nación y de numerosos organismos internacionales. En esta temática, aunque parezca irónico, la pandemia ha tenido un sesgo especial, ya que ha visibilizado un derecho que, para los sectores dirigentes y autoridades estaba oculto o solapado. Sin entrar en discusiones éticas, que a veces forman parte de posiciones ideológicas o políticas, el pedido constante de la apertura de servicios mínimos de las bibliotecas ha demostrado que estas son agentes indispensables -y de primera línea- para sostener e impulsar la diversidad en cualquier circunstancia. Pero su poder radica, como ha acontecido en el desarrollo de conflictos bélicos, desastres naturales, persecuciones y siniestros, en el hecho de que su presencia es indispensable como fenómeno social para la construcción de los deseos y los proyectos de vida de las personas en contextos de una amplia movilidad social.

Y la pospandemia acentuará la misión de las bibliotecas en su gestación e incremento de ciudadanía. Los bibliotecarios y bibliotecarias deberán apelar, nuevamente, a su imaginación para conciliar el acceso a los libros en forma presencial bajo el rigor de los protocolos sanitarios. En un sentido lato nos estamos reconvirtiendo en árbitros para determinar el grado de accesibilidad a nuestras bibliotecas. Es una situación delicada, de un gran cuidado entre la libertad de acceso y su restricción. Una dialéctica de difícil resolución, pero que brinda una gran oportunidad para demostrar a aquellos sectores que las tenían como instituciones desapercibidas o postergadas, que estas aún tienen mucho por decir y, lo que es más, por hacer en materia de inclusión.

Hay una constelación casi infinita de reflexiones y pensamientos acerca de la pandemia y su pospandemia. No es factible entonces -y menos nuestra intencionalidaddecir algo original o ya no dicho o escrito. Somos conscientes de que este Editorial es solo la fotografía de una situación determinada y que sus palabras pueden caducar rápidamente. Meditar sobre una realidad siempre implica el riesgo de su inmediata desactualización. Pero es posible pasar revista a algunos de los puntos reseñados en este texto: la adaptación de las bibliotecas a todo tipo de acontecimientos y fenómenos $y$, por ende, la ductilidad de las mentalidades profesionales para mantenerlas con vida en la sociedad; la necesidad no solo de pensar estas instituciones en el futuro sino, además, en nuestro "aquí y ahora", pues estas ya viven una "presentización del devenir"; el incremento, cada vez más notable, de ejercer la profesión en el horizonte expansivo y poroso de lo institucional en su íntima relación con la gestión remota doméstica; la construcción de un profesional fraccionado y suspendido en una realidad distante y fragmentada o facetada; la revaloración de otras esferas (o reformas) de la esencialidad presencial para no perder el contacto visual, dialogado y físico con los lectores y las lectoras; el advenimiento definitivo de los bibliotecarios y bibliotecarias como integrantes de los planteles de educación formal y la importancia del rol de las redes de bibliotecas para fortalecer este salto cualitativo; la defensa de lineamientos de agremiación para defender nuestros puestos de trabajo y los peligros de la "flexibilización laboral remota"; y la ocasión manifiesta que se nos presenta para ampliar los derechos de la gente que habían sido soslayados o velados o que se están generando 
en el ambiente pospandémico, y de este modo adelantarse a sus urgencias, entre otros numerosos aspectos.

Así la pospandemia puede impulsar a las bibliotecas hacia una perspectiva situacional enfocada en la demanda y la importancia de sus servicios como nunca antes lo habían tenido; un fenómeno que no sucedía desde el último tercio del siglo XIX cuando los procesos masivos de escolaridad y alfabetización sostuvieron y propiciaron la proliferación de las bibliotecas públicas. Todo desastre de crisis -las bibliotecas en estos momentos no son para nada la excepción- abren la oportunidad para reconfigurar los valores y las funciones de toda entidad, en una perspectiva indudablemente trágica pero, tal como se ha observado, que hace a la identidad de la resistencia y la supervivencia bibliotecaria.

¿Y ahora qué? Ya sabemos que la incertidumbre del porvenir, anclada en el ojo de tormenta del presente pospandémico, condicionará el conjunto del quehacer bibliotecario. Pero contra todo pronóstico, el cielo de las bibliotecas continuará brillando. Es muy conocida la opinión de Jorge Luis Borges en el libro Siete noches, cuando en su ensayo sobre "La poesía" cita un comentario de Ralph Waldo Emerson a propósito de la biblioteca como "un gabinete mágico", donde residen "muchos espíritus hechizados" (1989 [1980]: 254). No tenemos más que apelar a las herramientas que siempre han tenido estas entidades para desplegar, más que nunca, el abanico de posibilidades mágicas que irradia desde ellas y hacia sus futuras realizaciones. El "gabinete mágico" está esperando un diseño que antes no existía y solo las bibliotecarias y los bibliotecarios serán capaces de remodelarlo en la plenitud simbólica de su total significación. Un significado que también implica el hechizo por recuperar el día a día de las bibliotecas que vendrán. 


\section{Q Referencias bibliográficas}

"ABGRA. 2020. Bibliotecas argentinas: reflexiones, acciones y propuestas en tiempos de pandemia. La experiencia de la BCN, BNM, BNMM y CONABIP. Compilado y publicado por ABGRA; Editores: Jessica Soledad Castaño y Fernando Gabriel Gutiérrez Buenos Aires: Asociación de Bibliotecarios Graduados de la República Argentina.<http://abgra.org.ar/bibliotecas-argentinas-reflexiones-acciones-ypropuestas-en-tiempos-de-pandemia/> [Consulta: 03 marzo 2021].

"Arroyo-Vázquez, Natalia y José Antonio Gómez-Hernández. 2020. La biblioteca integrada en la enseñanza universitaria online: situación en España. En Profesional de la información. Vol. 29, no. 4, e290404. <https://doi.org/10.3145/ epi.2020.jul.04>

»Borges, Jorge Luis. 1989 [1980]. Siete noches. En Obras completas ${ }^{* *}:$ 1975-1985. Buenos Aires: Emecé, 1989. p. 254.

»Chartier, Roger. 2021. Lectura y pandemia. Conversaciones. Buenos Aires; Madrid: Katz Editores. (Discusiones).

"Connaway, Lynn Silipigni. 2020. The New Model Library. Welcome home. <https:// blog.oclc.org/next/the-new-model-library-welcome-home/> [Consulta: 21 marzo 2021].

"Gaitán, Anabel y María Inés Coraglia. 2021. Las bibliotecas universitarias argentinas y la educación en línea ante la pandemia por COVID19. En Anales de Documentación. Vol. 24, no. 1. <http://dx.doi.org/10.6018/analesdoc.439631>

"Gutiérrez, Fernando Gabriel y Jessica Castaño. 2020. Informe Bibliotecas argentinas ante el aislamiento social y obligatorio por el COVID-19. Buenos Aires: ABGRA. <https://abgra.org.ar/informe-bibliotecas-argentinas/> [Consulta: 28 enero 2021].

"Instituto de Investigaciones Bibliotecológicas. 2020. ¿Qué hacer desde las bibliotecas durante la pandemia? Ciclo de "Charlas del INIBI/9, miércoles 2 de diciembre de 2020. <https://www.youtube.com/watch?v=FgziY3lljio> [Consulta: 27 enero 2021].

"Las bibliotecas populares se reinventaron durante la pandemia, con delivery y diversas actividades. <https://www.telam.com.ar/notas/202009/514860-las-bibliotecas-publicas-se-reinventaron-durante-la-pandemia-con-delivery-y-diversas-actividades.html> [Consulta: 07 marzo 2021].

"Leer la ciudad. Bibliotecas en tiempos de crisis. 2020. <https://www.youtube. $\mathrm{com} /$ watch?v=SWO8E6URNol> [Consulta: 07 marzo 2021].

"Rodríguez, José Manuel. 2020. La biblioteca argentina que reparte libros puerta a puerta por el coronavirus. <https://www.efe.com/efe/america/cultura/la-biblioteca-argentina-que-reparte-libros-puerta-a-por-el-coronavirus/20000009-4214059> [Consulta: 06 marzo 2021].

"Suarez, Michel, 2020. Biblioteca Nacional Mariano Moreno. Las colecciones bibliográficas especiales: diferentes miradas. Robert Darnton y Michael Suarez. "Conferencias internacionales 2020" (agosto-septiembre 2020). BNMM. Min. $19.41<$ https://www.youtube.com/watch?v=ncUyy7mLyfg> [Consulta: o6 marzo 2021]. 